\title{
The renormalized jellium model for spherical and cylindrical colloids
}

Cite as: J. Chem. Phys. 126, 014702 (2007); https://doi.org/10.1063/1.2387168

Submitted: 12 July 2006 . Accepted: 10 October 2006 . Published Online: 03 January 2007

Salete Pianegonda, Emmanuel Trizac, and Yan Levin

\section{ARTICLES YOU MAY BE INTERESTED IN}

A self-consistent renormalized jellium approach for calculating structural and thermodynamic properties of charge stabilized colloidal suspensions

The Journal of Chemical Physics 131, 074115 (2009); https://doi.org/10.1063/1.3211305

Effective charge saturation in colloidal suspensions

The Journal of Chemical Physics 117, 8138 (2002); https://doi.org/10.1063/1.1511507

Charge renormalization, osmotic pressure, and bulk modulus of colloidal crystals: Theory

The Journal of Chemical Physics 80, 5776 (1984); https://doi.org/10.1063/1.446600

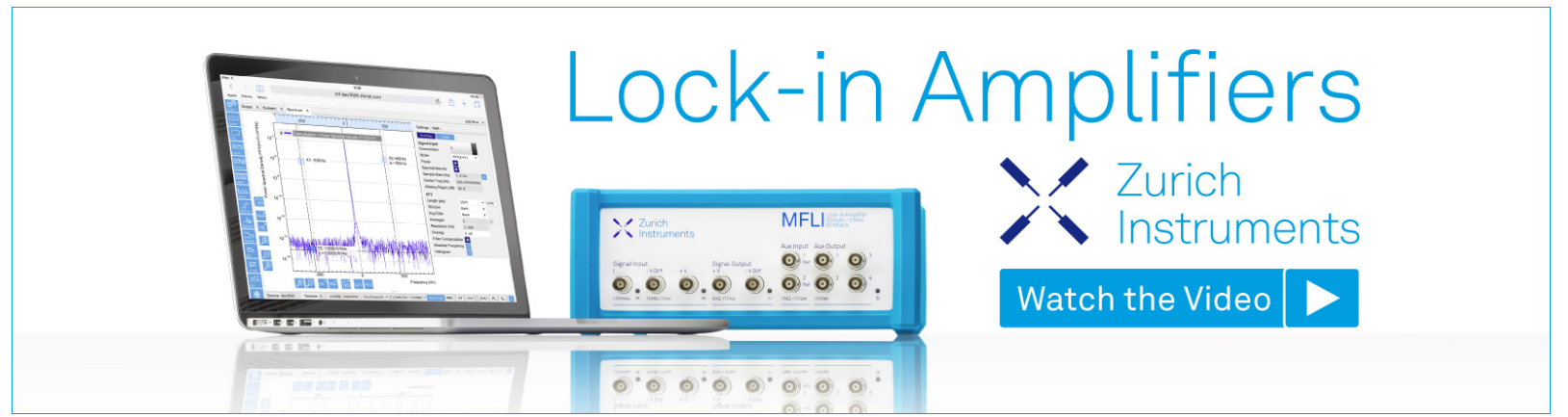

J. Chem. Phys. 126, 014702 (2007); https://doi.org/10.1063/1.2387168

(c) 2007 American Institute of Physics. 


\title{
The renormalized jellium model for spherical and cylindrical colloids
}

\author{
Salete Pianegonda \\ Laboratoire de Physique Théorique et Modèles Statistiques [Unité Mixte de Recherche (UMR) 8626 du \\ CNRS], Bâtiment 100, Université de Paris-Sud, 91405 Orsay Cedex, France and Instituto de Física, \\ Universidade Federal do Rio Grande do Sul, CP 15051, 91501-970 Porto Alegre (RS), Brazil \\ Emmanuel Trizac $^{\text {a) }}$ \\ CNRS, Université Paris-Sud, UMR 8626, LPTMS, F-91405, Orsay Cedex, France and Center for \\ Theoretical Biological Physics, UC San Diego, MC 0374, La Jolla, California 92093-0374 \\ Yan Levin \\ Instituto de Física, Universidade Federal do Rio Grande do Sul, CP 15051, 91501-970 Porto Alegre (RS), \\ Brazil
}

(Received 12 July 2006; accepted 10 October 2006; published online 3 January 2007)

\begin{abstract}
Starting from a mean-field description for a dispersion of highly charged spherical or (parallel) rodlike colloids, we introduce the simplification of a homogeneous background to include the contribution of other polyions to the static field created by a tagged polyion. The charge of this background is self-consistently renormalized to coincide with the polyion effective charge, the latter quantity thereby exhibiting a nontrivial density dependence, which directly enters into the equation of state through a simple analytical expression. The good agreement observed between the pressures calculated using the renormalized jellium and Monte Carlo simulations confirms the relevance of the renormalized jellium model for theoretical and experimental purposes and provides an alternative to the Poisson-Boltzmann cell model since it is free of some of the intrinsic limitations of this approach. () 2007 American Institute of Physics. [DOI: 10.1063/1.2387168]
\end{abstract}

\section{INTRODUCTION}

In the realm of soft matter, a quantitative description of systems with a nonvanishing density of mesoscopic constituents (colloids) is a difficult task whenever long range Coulomb interactions are present. ${ }^{1}$ It is customary to introduce a suitably defined Wigner-Seitz-type cell to render the situation tractable. This considerable simplification allows for the computation of various thermodynamic quantities (see, e.g., Refs. 2-5 for more recent accounts). Transport properties may also be derived. ${ }^{6,7}$ Initially borrowed from solid state physics, the concept of a cell nevertheless appears fruitful to describe the phase behavior of liquid phases (see, e.g., Ref. 8). When a more microscopic information such as effective interaction is sought, there is, however, to date no evidence that the cell picture provides accurate answers, through the approximate procedures that have been proposed to infer solvent and microion averaged intercolloid potentials of interaction. ${ }^{3}$ In the present paper, we adopt a more "liquidstate" viewpoint to describe the local and global screening properties of microscopic ions around highly charged rodlike or spherical colloids, taking due account of the finite density of colloids. Our approach bears a strong resemblance with a jellium model put forward by Beresford-Smith et al. ${ }^{10}$ with the important difference that the jellium under consideration here is the "renormalized" counterpart of that studied in Ref. 10. A preliminary account with emphasis on the sedimenta-

${ }^{a)}$ Electronic mail: trizac@1ptms.u-psud.fr tion of charged colloids has been published in Ref. 11 and we note that our approach has been recently tested with some success on liposome dispersions. ${ }^{12-14}$

The article is organized as follows. The model is defined in Sec. II and illustrated in Sec. III where salt-free suspensions are considered and the numerical procedure exemplified with charged spheres. The cylindrical geometry is also addressed which allows to discuss the fate of the classical Manning-Oosawa condensation phenomenon ${ }^{15,16}$ within the present framework. The effects of an added electrolyte are investigated in Sec. IV and concluding remarks are presented in Sec. V. As will become clear below, our framework provides a procedure to incorporate self-consistently charge renormalization into the classical Donnan equilibrium description of suspensions.

\section{THE MODEL}

When immersed in a polar solvent, mesoscopic "particles" release small ions in the solution that, together with other microions resulting from the dissociation of an added salt, form an inhomogeneous cloud around each colloid. Within the mean-field approximation to which we restrict here, neglect of microionic correlations allows to relate the local density of microspecies of valency $z_{i}$ at point $\boldsymbol{r}$ to the local electrostatic potential $\varphi(\boldsymbol{r})$ through $n_{i}(\boldsymbol{r})$ $\propto \exp \left(-z_{i} e \beta \varphi(\boldsymbol{r})\right)$, where $e$ is the elementary charge and $\beta^{-1}=k T$ is the thermal energy. ${ }^{1,17}$ For any position of the $N$ colloids present, one needs to solve the resulting PoissonBoltzmann equation from which the electric potential follows. This potential may then be inserted into the stress 
tensor ${ }^{1}$ to compute the force acting on the colloids. Such a procedure, which makes explicit use of the separation of time scales between colloids and microions, opens the way towards a complete description of the statics and dynamics of the system, with, e.g., Monte Carlo or molecular dynamics techniques to treat the colloidal degrees of freedom. ${ }^{18,19}$ This treatment is, however, numerically particularly demanding and much insight is gained from further approximations that map the original problem with $N$ colloids onto a one-colloid situation. The cell model is an option, where the finite density of colloids is accounted for by an exclusion region. We propose here an alternative, free of some of the limitations of the cell model, that is equally simple to implement and solve.

A given colloid with bare charge $Z_{\mathrm{bare}} e$, assumed positive, is tagged and fixed at the origin. The suspending medium (solvent treated as a dielectric continuum with permittivity $\varepsilon$ ) is taken as infinite, with a mean colloidal density $\rho$. Following Ref. 10, the colloids around the tagged particle are assumed to form a homogeneous background, with charge density $Z_{\text {back }} e \rho$, so that the electrostatic potential around the tagged colloid fulfills Poisson's equation

$$
\nabla^{2} \varphi=-\frac{4 \pi}{\varepsilon}\left[Z_{\mathrm{back}} \rho e+\sum_{i} n_{i}^{0} z_{i} e e^{-\beta e z_{i} \varphi}\right]
$$

where the summation runs over all microspecies and the concentrations $n_{i}^{0}$ are determined either from electroneutrality in the no salt case or from the osmotic equilibrium with a salt reservoir in the semi-grand-canonical situation, both addressed here. At large distances $(r \rightarrow \infty)$, the term in brackets on the right hand side of (1) vanishes which imposes a value $\varphi_{\infty}$ for the potential far from the colloid, that may be called a Donnan potential. The key point in our approach is that unlike in Ref. $10, Z_{\text {back }} \neq Z_{\text {bare }}$ : the background charge is not known a priori but is determined self-consistently as explained below.

To illustrate the methodology, we consider a spherical colloid of radius $a$. When $r \rightarrow \infty$, we may linearize (1) around $\varphi_{\infty}$, which results in a Helmholtz equation indicating that

$$
\varphi(\boldsymbol{r}) \stackrel{r \rightarrow \infty}{\sim} \varphi_{\infty}+\frac{Z_{\mathrm{eff}} e}{\varepsilon(1+\kappa a) r} e^{-\kappa(r-a)},
$$

where the characteristic distance $\kappa^{-1}$ is given by

$$
\kappa^{2}=4 \pi \sum_{i} \frac{\beta e^{2}}{\varepsilon} n_{i}^{0} z_{i}^{2} e^{-\beta e z_{i} \varphi_{\infty}}=4 \pi \ell_{B} \sum_{i} z_{i}^{2} n_{i}(\infty)
$$

and $l_{B}=\beta e^{2} / \varepsilon$ is the Bjerrum length.

For very low bare charges, solution (2) holds for all distances with $Z_{\text {eff }}=Z_{\text {bare }}$, and one can consider that $Z_{\text {back }}$ $=Z_{\text {bare }}$. However, typical colloidal charges are such that $Z_{\mathrm{bare}} l_{B} / a \gg 1$, a regime for which counterions become strongly associated with the colloid and the charge renormalization effects ${ }^{20-22}$ cannot be ignored. The counterion condensation strongly affects the electrostatic far field so that the large distance signature involves an effective charge $\left[Z_{\text {eff }}\right.$ in Eq. (2)] which significantly differs from the bare one. As a result of nonlinear screening, one has $Z_{\text {eff }} \ll Z_{\text {bare }}$ whenever $Z_{\text {bare }} l_{B} / a \gg 1$.
At this point, the effective charge arising in (2) is a function of both the background and the bare charge, other parameters being fixed: $Z_{\mathrm{eff}}=Z_{\mathrm{eff}}\left(Z_{\mathrm{back}}, Z_{\mathrm{bare}}\right)$. As far as a static description is pursued, for sufficiently strongly charged colloids the bare charge is an irrelevant quantity far enough from the tagged colloid, and we demand that $Z_{\text {back }}$ coincides with $Z_{\text {eff }}$, which best characterizes the background charge resulting from smearing out the other colloid contributions. We therefore enforce the self-consistency constraint

$$
Z_{\text {back }}=Z_{\text {eff }}\left(Z_{\text {back }}, Z_{\text {bare }}\right)
$$

to compute the a priori unknown background charge. As we shall see below, this condition is readily implemented numerically and for a given $Z_{\text {bare }}$ leads to a unique value for $Z_{\text {back }}=Z_{\text {eff }}$. This value is density dependent, which is also the case of the inverse screening length $\kappa$. Indeed, $\varphi_{\infty}$ depends on $\rho$ (Ref. 23) through the electroneutrality condition

$$
Z_{\mathrm{back}} \rho e+\sum_{i} n_{i}^{0} z_{i} e \exp \left(-\beta e z_{i} \varphi_{\infty}\right)=0,
$$

which translates into a $\rho$ dependence for $\kappa$. Considering now two colloids in the weak overlap approximation (i.e., not too close), the effective potential of interaction will take a (DLVO) Derjaguin, Landau, Verwey, Overbeek form ${ }^{1,17}$ with effective parameters $\kappa$ and $Z_{\text {eff }}$.

The procedure outlined here incorporates nonlinear screening together with finite $\rho$ effects. It is best suited to describe low density systems since the colloid-colloid pair distribution function $g_{c c}$ is implicitly considered to be unity for all distances. This reduction, which has nontrivial consequences, is certainly of little relevance for high density suspensions for which the cell model is presumably a better approximation.

Before illustrating the method, we briefly consider the pressure in the system, that is given by the densities of microions far from the tagged colloid:

$$
\beta P=\sum_{i} n_{i}(\infty)=\sum_{i} n_{i}^{0} \exp \left(-\beta e z_{i} \varphi_{\infty}\right) .
$$

The colloidal contribution is explicitly discarded. ${ }^{11}$ This is well justified in the low salt limit, which is a regime of counterion dominance provided that $Z_{\mathrm{bare}} \gg 1$, which is easily achieved in practice.

\section{THE NO SALT LIMIT}

\section{A. Spherical colloids}

The simplest situation to investigate is that of de-ionized suspensions (no salt). For simplicity, we consider counterions as monovalent. From (1) it follows that the dimensionless potential $\phi=\beta e \varphi$ obeys the equation

$$
\frac{d^{2} \phi}{d \widetilde{r}^{2}}+\frac{2}{\widetilde{r}} \frac{d \phi}{d \widetilde{r}}=3 \eta \frac{Z_{\mathrm{back}} \ell_{B}}{a}\left(e^{\phi}-1\right),
$$

where $a$ is again the radius of the tagged particle from which the dimensionless distance $\tilde{r}=r / a$ is defined and $\eta$ $=4 \pi \rho a^{3} / 3$ is the volume fraction. The boundary conditions are 


$$
\begin{aligned}
& \phi \rightarrow 0 \text { for } \tilde{r} \rightarrow \infty, \\
& \frac{d \phi}{d \tilde{r}}=-\frac{Z_{\text {bare }} \ell_{B}}{a} \text { for } \tilde{r}=1 .
\end{aligned}
$$

In writing (7), use has been made of the (global) electroneutrality constraint $n_{-}^{0} e \exp \left(\phi_{\infty}\right)=Z_{\text {back }} \rho e$ with the choice $\phi_{\infty}$ $=0$. For all values of $Z_{\text {back }}$, the far field of $\phi$ is governed by $\kappa$ such that

$$
(\kappa a)^{2}=3 \eta \frac{Z_{\mathrm{back}} \ell_{B}}{a}
$$

The above system is solved following similar lines as in Ref. 24. We summarize here the main steps. In practice, Eq. (7) is solved numerically for a finite system $\widetilde{r} \in[1, \widetilde{R}]$, where $\widetilde{R}$ needs to be large enough (that is, $\kappa a \widetilde{R} \gg 1$, but note that $\kappa$ is not known initially but follows once the background charge is known). (a) The first and important step is to rephrase the boundary value problem at hand as an initial value problem with boundary conditions $\phi^{\prime}(\widetilde{R})=0$ (to ensure electroneutrality) and varying $\phi(\widetilde{R})$. The volume fraction $\eta$ is fixed and the background charge $Z_{\text {back }}$ first assigned a guess value, to be modified iteratively (see below). If $\phi(\widetilde{R})$ is small enough, the system then admits a solution. (b) From this solution, one computes $\phi^{\prime}(\widetilde{r}=1)$ to know the corresponding bare charge. (c) Changing $\phi(\tilde{R}),{ }^{25}$ the targeted value $\phi^{\prime}(\tilde{r}$ $=1)=-Z_{\text {bare }} \ell_{B} / a$ is readily found by iteration. (d) The screening quantity $\kappa$ is subsequently computed from (10) and the effective charge associated with the particular couple $\left(Z_{\text {back }}, Z_{\text {bare }}\right)$ is deduced from the large $\tilde{r}$ behavior of $\phi$ (e.g., one needs to observe a clear-cut plateau for $[\phi(\widetilde{r})$ $-\phi(\widetilde{R})] e^{\kappa a \tilde{r}} \tilde{r}$ plotted as a function of $\tilde{r}$ in the range $1 \ll \tilde{r}$ $<\widetilde{R})$. The first iteration ends here, and the procedure is repeated with the $Z_{\text {eff }}$ obtained as the next trial value for $Z_{\text {back }}$. Alternatively, one may sample several trial values for $Z_{\text {back }}$

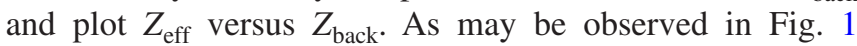
where such a plot is displayed, the dependence of $Z_{\text {eff }}$ on $Z_{\text {back }}$ is very mild, which means that convergence towards $Z_{\text {back }}=Z_{\text {eff }}$ is achieved in a few steps. In the (artificial) limit where $Z_{\text {back }} \rightarrow 0$, the problem at hand reduces to an unscreened one (governed by Laplace equation) with solution $\phi=Z_{\text {bare }} \ell_{B} / r$ : there is therefore no renormalization of effective charge so that $Z_{\text {eff }} \rightarrow Z_{\text {bare }}$ (see Fig. 1). The inset shows how the self-consistent background charge is determined, the other points being unphysical.

In the limit of a diverging bare charge, the procedure is well behaved and yields a finite self-consistent effective charge. From the previous discussion, we expect $Z_{\text {eff }}$ to diverge at small $Z_{\text {back}}$, which is indeed the case (not shown).

Once the physical solution to the problem has been located (inset of Fig. 1), various quantities such as the pressure may be computed. In the remainder, we will use the terms "effective" and "background" charges to refer to the selfconsistent solution as obtained in Fig. 1: $Z_{\text {eff }}=Z_{\text {back }}$ is therefore a function of $Z_{\text {bare }}$ and volume fraction (possibly also salt concentration, see Sec. IV). For a particular density, this function is shown in Fig. 2. After the initial linear regime,

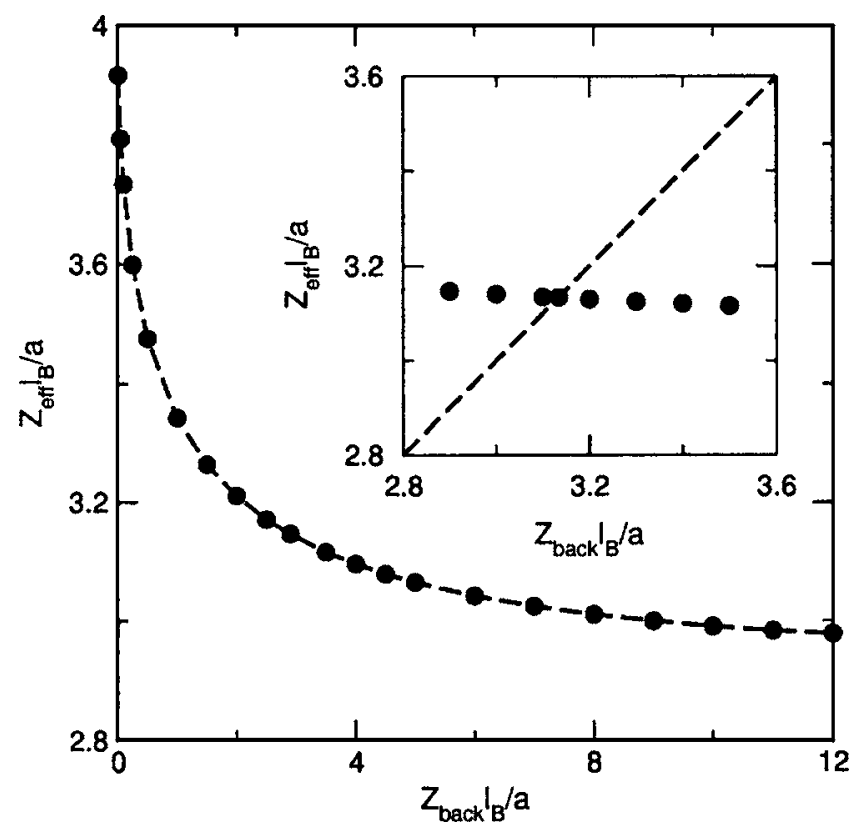

FIG. 1. The effective charge as a function of background charge for $\eta$ $=10^{-2}$ and $Z_{\text {bare }} \ell_{B} / a=4$ (spherical colloids, no salt). The physical solution $Z_{\text {eff }}=Z_{\text {back }}$ to the problem is the point of intersection with the first bisectrix (see inset, where a magnification of the relevant part of the main graph is shown).

where no renormalization takes place, the effective charge slowly reaches a saturation plateau as $Z_{\text {bare }} \rightarrow \infty$. For this specific density $\left(\eta=10^{-2}\right)$ the effective charge saturates at $Z_{\text {eff }} \ell_{B} / a \simeq 6.6$. The saturation phenomenon observed here is strongly reminiscent of that observed in the classical Poisson-Boltzmann approach (either in a cell or in an infinite medium $\left.^{3,22,26}\right)$. To assess quantitatively the possible difference with the results obtained within the cell model, we compare in Fig. $3 Z_{\text {eff }}$ derived in the cell ${ }^{3,24}$ to its renormalized

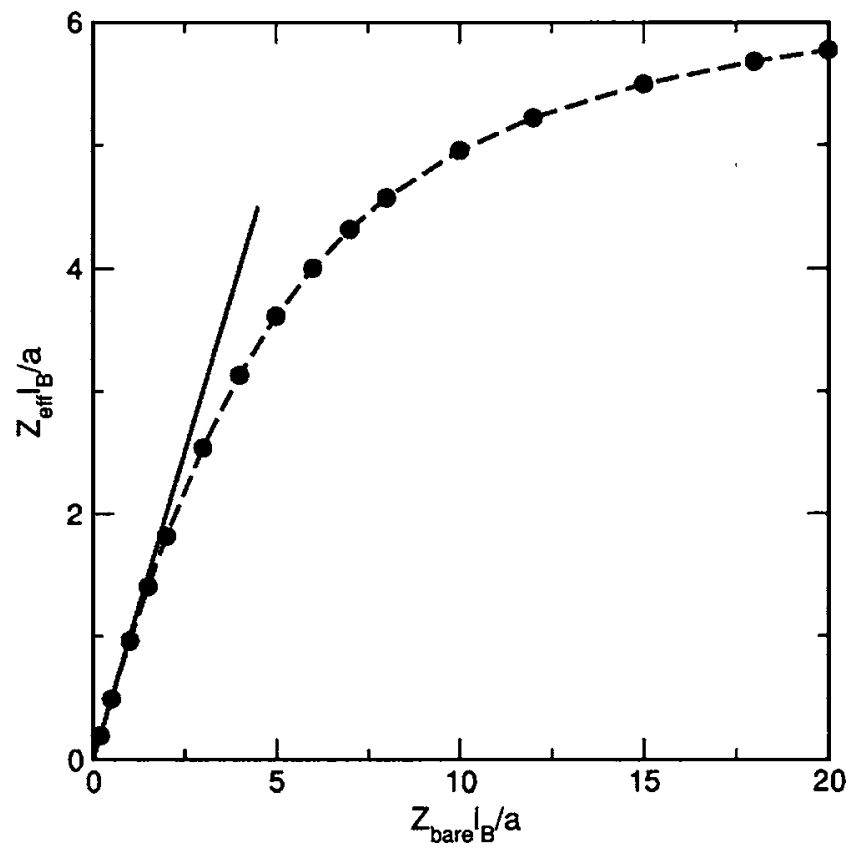

FIG. 2. The effective charge (or equivalently, the background charge) as a function of bare charge for $\eta=10^{-2}$ (spherical colloids, no salt). The line has slope unity to emphasize the initial "Debye-Hückel" regime. 


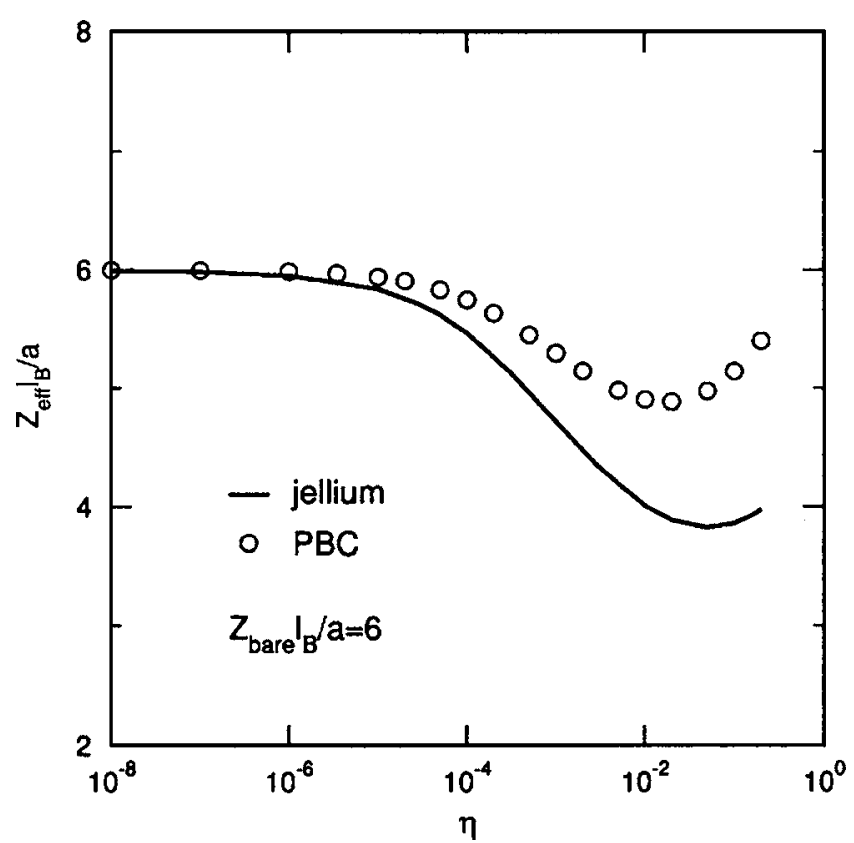

FIG. 3. Comparison between the effective charges within the PoissonBoltzmann cell (PBC) and the renormalized jellium model as a function of the volume fraction. Here $Z_{\mathrm{bare}} \ell_{B} / a=6$ (spherical colloids, no salt).

jellium counterpart. Both charges differ by a notable amount for $\eta>10^{-3}$ while the agreement at very low density is meaningless and follows from a divergence of the saturation effective charge in both models: nonlinear effects disappear when $\eta \rightarrow 0$, so that $Z_{\text {eff }} \rightarrow Z_{\text {bare }}$. This is a peculiarity of systems with colloidal spheres and counterions only, and it turns out that the behavior of charged cylinders is quite different, see below.

Under the de-ionized conditions studied here, the pressure takes the simple form $\beta P=Z_{\text {eff }} \rho$, whereas the corresponding expression in the cell model is less explicit and does not directly involve the effective charge. Remarkably, although there is a significant difference between the effective charges calculated within the Poisson-Boltzmann cell (PBC) model and the renormalized jellium, the pressures calculated using the two models are identical for $\eta<0.1$, see Fig. 4. A similar agreement is found at saturation. ${ }^{11}$ We add here that a comparison between the renormalized jellium equation of state and the "exact" results of the primitive model obtained using the Monte Carlo computations has been reported in Ref. 11, with excellent agreement (the corresponding density range is quite low such that $\mathrm{PBC}$ and renormalized jellium predictions agree).

Before concluding this section, we emphasize that one must carefully check that the results obtained do not depend on the particular value chosen for the cutoff $\widetilde{R}$, e.g., by repeating the analysis with an increased cutoff.

\section{B. Cylindrical colloids}

Consider a nematic phase of parallel infinite rods $(L$ $\rightarrow \infty$ ) with bare line charge density $\lambda_{\text {bare }}$ (therefore no positional order in the plane perpendicular to the main axis). We may repeat the previous approach, tagging a given rod of radius $a$ and modeling the effects of the other rods by a

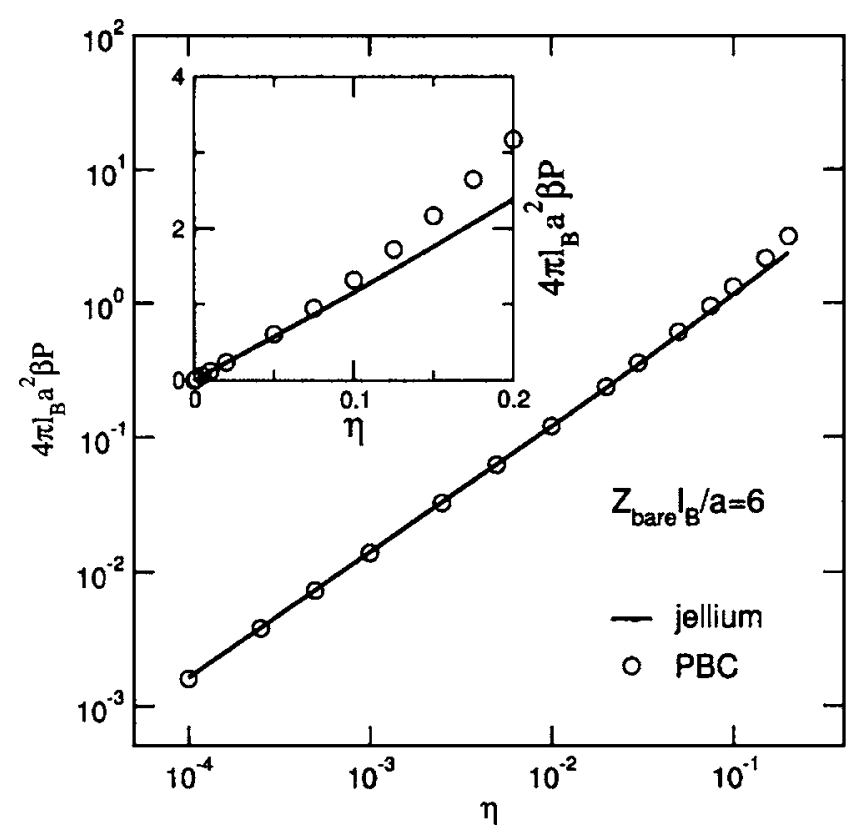

FIG. 4. Pressure as a function of volume fraction within the cell and the renormalized jellium model, on a log-log scale. Here, $Z_{\text {bare }} l_{B} / a=6$ (spherical colloids, no salt). The inset shows the same data on a linear scale.

homogeneous background, with line charge $\lambda_{\text {back }}$. While the definition of $\kappa$ is unaffected compared to the spherical case, the far-field potential now takes the form

$$
\phi(\widetilde{r})=\phi_{\infty}+2 \lambda_{\mathrm{eff}} \ell_{B} \frac{K_{0}(\kappa a \widetilde{r})}{\kappa a K_{1}(\kappa a)},
$$

where $K_{0}\left(K_{1}\right)$ denotes the zeroth (first) order modified Bessel function of the second kind. In the spirit of the consistency requirement of Sec. II, we impose $\lambda_{\text {back }}=\lambda_{\text {eff }}$ where again $\lambda_{\text {eff }}$ follows from the large distance behavior of the solution to Poisson's equation with background charge $\lambda_{\text {back }}$ :

$$
\frac{d^{2} \phi}{d \tilde{r}^{2}}+\frac{1}{\tilde{r}} \frac{d \phi}{d \widetilde{r}}=4 \eta \lambda_{\mathrm{back}} \ell_{B}\left(e^{\phi}-1\right) .
$$

Here the volume fraction is $\eta=\pi a^{2} n_{2 \mathrm{D}}$ where $n_{2 \mathrm{D}}$ is the mean surface density of rods (in the plane perpendicular to their axis). The boundary conditions are the same as (8) and (9) and the numerical method identical to that used in the spherical case.

The effective charges calculated using the cell and the renormalized jellium models are compared in Fig. 5 for $\lambda_{\text {bare }} \ell_{B}=1$. The inset corresponds to the saturation regime where $\lambda_{\text {bare }}$ is very large $\left(\lambda_{\text {bare }} \rightarrow \infty\right)$. We observe a substantial disagreement between the two effective charges. On the other hand, in the small bare charge regime where nonlinear effects are not at work, both quantities coincide (not shown), which is a signature of whenever nonlinear effects come into play (i.e., outside the small bare charge linear regime, which is the case for both figures). Beyond these differences, Manning-Oosawa condensation, ${ }^{15,16}$ which is a key feature of two-dimensional (2D) electrostatics, is shared by both PBC and renormalized jellium models. As the colloid density is decreased $\left(\eta \rightarrow 0^{+}\right)$, the effective charge becomes independent of the bare one, provided $\lambda_{\text {bare }}$ exceeds the critical 


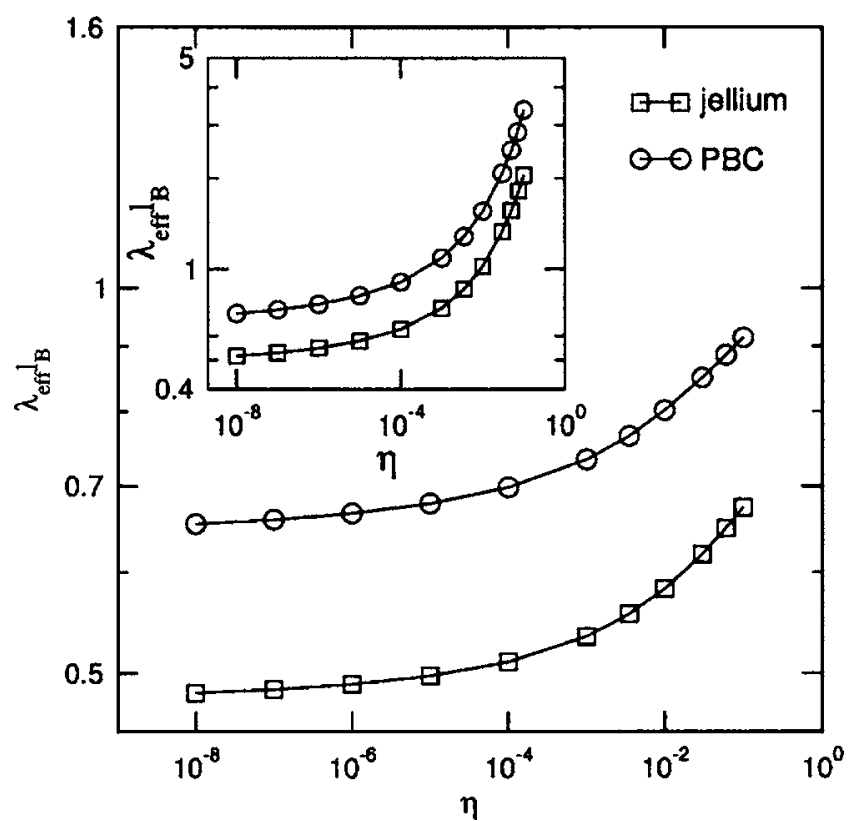

FIG. 5. Effective charge as a function of volume fraction within the PBC and the renormalized jellium model for $\lambda_{\text {bare }} \ell_{B}=1$. The inset corresponds to the saturation regime where $\lambda_{\text {bare }} \rightarrow \infty$ (rodlike colloids, no salt).

threshold $1 / \ell_{B}$. This feature is illustrated in Fig. 6. At the saturation plateau and again for $\eta \rightarrow 0^{+}$, one has $\lambda_{\text {eff }} \ell_{B}$ $\simeq 0.47$, a value that will be refined below.

To be more quantitative, it is furthermore natural to compare the corresponding functional forms of effective charges versus bare ones, and versus density in both $\mathrm{PBC}$ (where it can be computed analytically) and renormalized jellium models, where this information is accessed numerically. To this end, we reconsider the analytical results obtained in

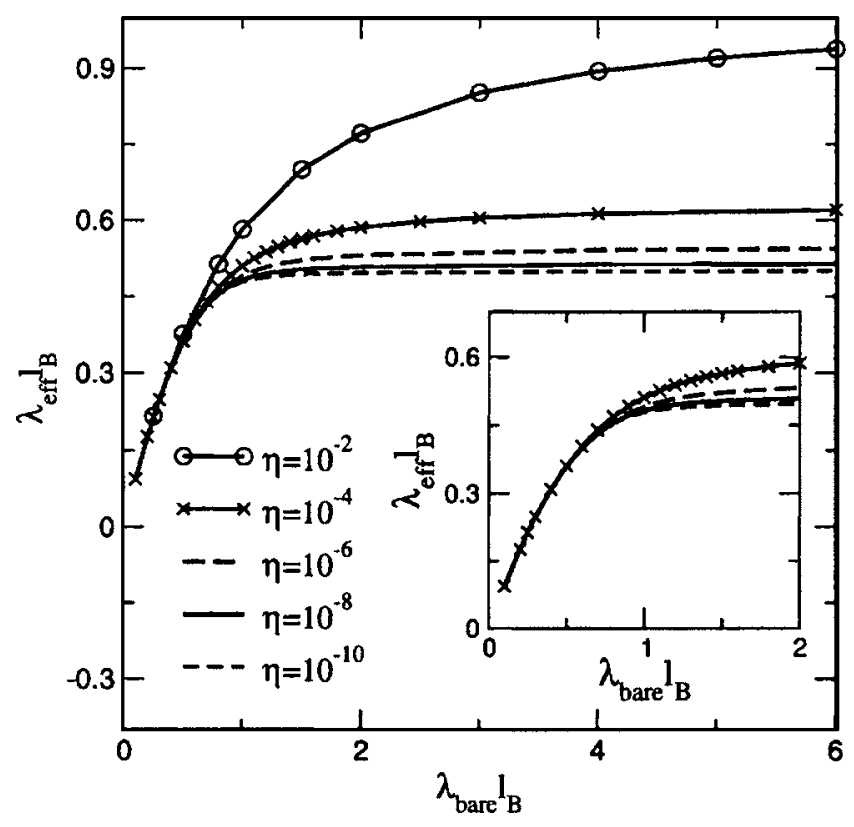

FIG. 6. The effective charge as a function of the bare charge for different values of volume fractions (renormalized jellium model for charged rods). The inset shows a magnification of the main graph in the low charge regime. The present scenario is exactly that of the Manning-Oosawa counterion condensation occurring in the cell model.
Refs. 22 and 24 where the effective charge in the cell model following the prescription of Alexander et $a l^{3}{ }^{3}$ reads

$$
\begin{aligned}
\lambda_{\mathrm{eff}} \ell_{B}= & \frac{1}{2} K_{\mathrm{PB}}^{2} a R_{w s}\left\{I_{1}\left(K_{\mathrm{PB}} R_{w s}\right) K_{1}\left(K_{\mathrm{PB}} a\right)\right. \\
& \left.-I_{1}\left(K_{\mathrm{PB}} a\right) K_{1}\left(K_{\mathrm{PB}}\right) R_{w s}\right\},
\end{aligned}
$$

with standard notation for the Bessel functions. Here $R_{w s}$ $\equiv a \eta^{-1 / 2}$ is the radius of the cell and $K_{\mathrm{PB}}$ is the inverse screening length related to the microionic density at the cell boundary, ${ }^{22}$ which can be computed explicitly from the analytical solution of Ref. 2. After some algebra, we find, to leading order in density, that when $\lambda_{\text {bare }}>1 / \ell_{B}$,

$$
\lambda_{\mathrm{eff}}^{\mathrm{sat}} \ell_{B} \stackrel{n \rightarrow 0^{+}}{\sim} \frac{\sqrt{2}}{2} I_{1}(\sqrt{2})+\pi^{2} \frac{I_{0}(\sqrt{2})+\sqrt{2} I_{1}(\sqrt{2})+I_{2}(\sqrt{2})}{(2 \xi-\log (\eta))^{2}},
$$

where $\xi=\lambda_{\text {bare }} /\left(\lambda_{\text {bare }}-1 / \ell_{B}\right)$. We note that the leading term $\sqrt{2} I_{1}(\sqrt{2}) / 2 \simeq 0.63$ differs from the value found in the renormalized jellium ( $\simeq 0.47$, see Fig. 6). Moreover, Eq. (14) also suggests a fitting form to describe the saturation plateau in the low density regime of the renormalized jellium model:

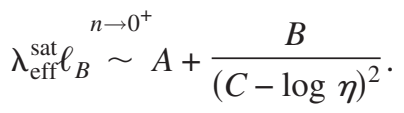

The values of $A, B$, and $C$ can be obtained using a numerical fit. We find that in the saturation limit $A \simeq 0.471, B \simeq 16.87$, and $C \simeq 0.843$ give an excellent agreement with the numerical data. We have also checked that an equally good agreement is found at lower bare charges, such as $\lambda_{\text {bare }} \ell_{B}=4$, but with different values of $A, B$, and $C$. We conclude here that both models are described by the same limiting law for low densities, at least beyond the condensation threshold.

It is of interest to resolve the condensate structure once the counterion condensation has set in. A useful measure of the condensate thickness is provided by the so-called Manning radius $R_{M}$ (Ref. 27) that has been recently worked out in the infinite dilution limit and for low salt content: ${ }^{16,28}$ in practice, the integrated charge per unit length $q(r)$ around a rod has an inflection point at $r=R_{M}$ when plotted as a function of $\log r$. This is exactly the point where $q\left(R_{M}\right) \ell_{B} / e=1$. We expect a similar behavior for the renormalized jellium, given that in the vicinity of highly charged rods, the (largely dominant) counterion distribution should not be sensitive to the difference between a uniform background as in the renormalized jellium model and coions as in the situation worked out in Ref. 16. The lower inset of Fig. 7 shows that this is indeed the case. In addition, from the analytical expressions derived in Ref. 16 and the fact that the relevant screening parameter reads here as $(\kappa a)^{2}=4 \eta \lambda_{\mathrm{eff}} \ell_{B}$, we expect the scaling $\kappa R_{M} \propto(\kappa a)^{1 / 2}$; more precisely

$$
R_{M} \stackrel{n \rightarrow 0^{+}}{\propto} a \eta^{-1 / 4} \exp \left(-\frac{1}{2\left(\lambda_{\text {bare }} \ell_{B}-1\right)}\right) .
$$

The dependence of $R_{M}$ on both density and bare charge embodied in Eq. (16) is fully supported by the numerical data, see Fig. 7.

Finally, and much like for spherical colloids, there is a good agreement between the osmotic pressure calculated us- 


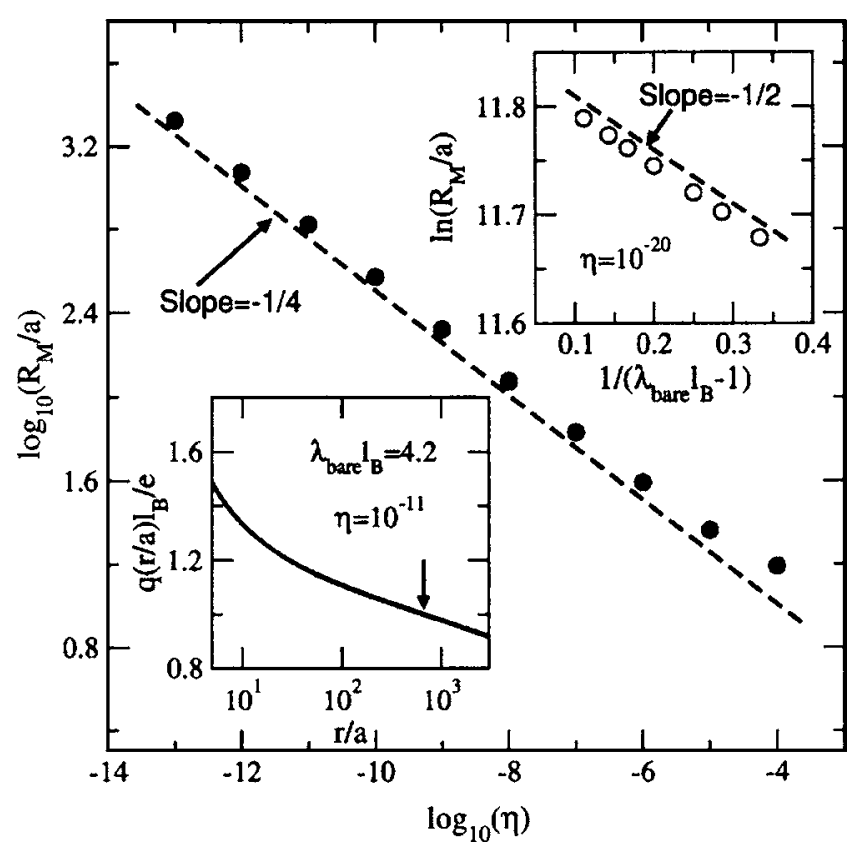

FIG. 7. Manning radius $R_{M}$ vs packing fraction for $\lambda_{\text {bare }} \ell_{B}=4$.2. Extremely low densities have been considered to see the predicted power law dependence $R_{M} \propto \eta^{-1 / 4}$, see Eq. (16). The upper inset shows that the bare charge dependence of $R_{M}$ also follows the form given by Eq. (16). The lower inset shows $q(\widetilde{r}) \ell_{B} / e$ as a function of distance from the rod axis on a linear-log scale: as expected, the inflection point, indicated by the vertical arrow, coincides with the point where $q\left(\widetilde{R}_{M}\right) \ell_{B} / e=1$.

ing the cell model and the renormalized jellium approximation, in spite of the different effective charges, see Fig. 8. Discrepancies are observed only for volume fractions $\eta$ $>0.06$ and the agreement seems to be better at high charges.

\section{EFFECTS OF ADDED SALT}

In this section, we consider systems dialyzed against an electrolyte reservoir with the monovalent salt concentration

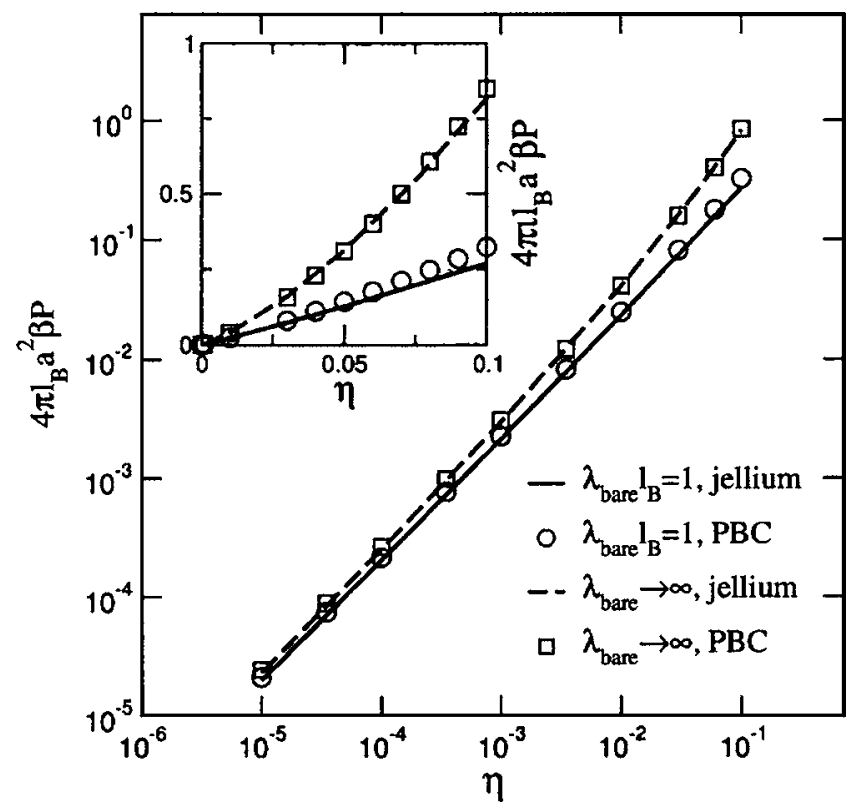

FIG. 8. Pressure as a function of volume fraction within the $\mathrm{PBC}$ and the renormalized jellium model for both a moderately charged and a highly charged rod (saturation limit), without added salt. The inset shows the same data on a linear scale. $c_{s}$. The corresponding screening parameter is $\kappa_{\mathrm{res}}^{2}=8 \pi \ell_{B} c_{s}$. It is convenient to choose the reference potential so that microionic densities are $n_{ \pm}(\boldsymbol{r})=c_{s} \exp [\mp \phi(\boldsymbol{r})]$, where the counterions are assumed to be monovalent. Using Eq. (5), the potential at infinity becomes

$$
\phi_{\infty}=\operatorname{arcsinh}\left(\frac{Z_{\mathrm{back}} \rho}{2 c_{s}}\right) .
$$

It is important to keep in mind that $n_{ \pm}(\boldsymbol{r})$ are not the physical microion densities but are the effective (renormalized) quantities satisfying

$$
\int d \boldsymbol{r}\left[n_{+}(\boldsymbol{r})-n_{-}(\boldsymbol{r})+Z_{\mathrm{eff}} \rho\right] \equiv-Z_{\mathrm{bare}} .
$$

Since the renormalization does not affect coions, their concentration inside the jellium with one colloid fixed at $r=0$ is

$$
C_{+}=\frac{1}{V} \int d \boldsymbol{r} n_{+}(\boldsymbol{r}),
$$

where it is understood that $V$ denotes the measure of a large volume centered at $\boldsymbol{r}=0$. The concentration of counterions, $C_{-}$, then follows from the overall charge neutrality inside suspension, $C_{-}=C_{+}+Z_{\text {bare }} \rho$.

Far from the colloid, $n_{+}(\boldsymbol{r})$ saturates at the bulk value $\tilde{n}_{+}$, so that in the thermodynamic limit $(V \rightarrow \infty)$

$$
C_{+}=\tilde{n}_{+} .
$$

Similarly, for $V \rightarrow \infty$

$$
\frac{1}{V} \int d \boldsymbol{r}\left[n_{+}(\boldsymbol{r})-n_{-}(\boldsymbol{r})+Z_{\mathrm{eff}} \rho\right]=\frac{-Z_{\mathrm{bare}}}{V} \rightarrow 0,
$$

which means that

$$
C_{+}-\frac{1}{V} \int d \boldsymbol{r} n_{-}(\boldsymbol{r})+Z_{\mathrm{eff}} \rho=0 .
$$

The charge neutrality allows us to rewrite Eq. (22) as

$$
\frac{1}{V} \int d \boldsymbol{r} n_{-}(\boldsymbol{r})=C_{-}-\left(Z_{\text {bare }}-Z_{\text {eff }}\right) \rho .
$$

Equation (23) provides a suggestive interpretation of $n_{-}(\boldsymbol{r})$ as the local density of free (uncondensed) counterions. Far from the colloid, $n_{-}(\boldsymbol{r})$ saturates at its bulk value $\widetilde{n}_{-}$, and in the thermodynamic limit Eq. (23) reduces to

$$
C_{-}=\tilde{n}_{-}+\left(Z_{\text {bare }}-Z_{\text {eff }}\right) \rho .
$$

Equations (20) and (24) allow us to calculate the ionic content inside a suspension dialyzed against a salt reservoir. This is particularly useful when comparing the results of the renormalized jellium model, which is grand canonical in electrolyte, with the Monte Carlo simulations, which are usually performed in a canonical ensemble. Knowledge of the asymptotic potential allows us to obtain the concentrations of coions and free counterions inside the suspension,

$$
\tilde{n}_{ \pm}=c_{s} \exp \left(\mp \phi_{\infty}\right) .
$$

These are precisely the densities that govern screening within the renormalized jellium, $\kappa^{2}=4 \pi \ell_{B}\left(\widetilde{n}_{+}+\widetilde{n}_{-}\right)$. 


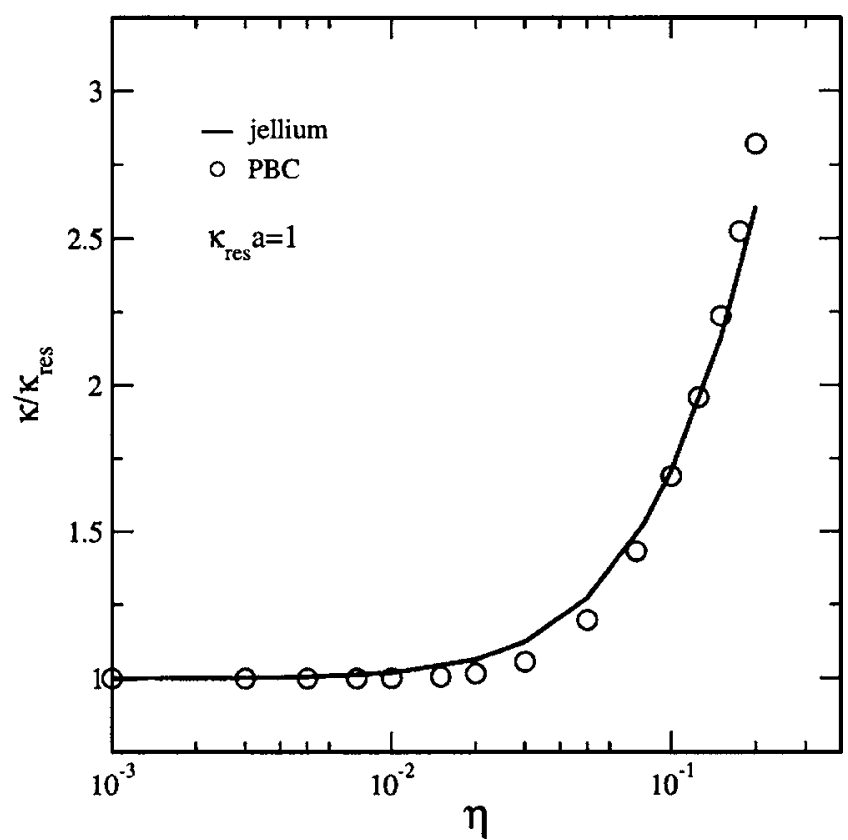

FIG. 9. Ratio between $\kappa$ and $\kappa_{\text {res }}$ as a function of volume fraction for $\kappa_{\text {res }} a=1$ (saturation regime and spherical colloids).

\section{A. Spherical colloids}

In spherical geometry, Poisson equation (1) now takes the form

$$
\frac{d^{2} \phi}{d \widetilde{r}^{2}}+\frac{2}{\tilde{r}} \frac{d \phi}{d \widetilde{r}}=\left(\kappa_{\mathrm{res}} a\right)^{2} \sinh \phi-3 \eta \frac{Z_{\mathrm{back}} \ell_{B}}{a} .
$$

We again solve it numerically as a boundary value problem in a (large enough) finite cell with vanishing $\phi^{\prime}$ at the boundary, increasing gradually the boundary potential from the value

$$
\phi_{\infty}=\operatorname{arcsinh}\left[\frac{3 \eta Z_{\mathrm{back}} \ell_{B} / a}{\left(\kappa_{\mathrm{res}} a\right)^{2}}\right],
$$

which corresponds to a vanishing bare charge.

Linearizing Eq. (26) around $\phi_{\infty}$, it can be seen that at large distances the potential takes the form of Eq. (2), with a screening constant $\kappa$ given by

$$
(\kappa a)^{4}=\left(\kappa_{\mathrm{res}} a\right)^{4}+\left(\frac{3 \eta Z_{\mathrm{back}} \ell_{B}}{a}\right)^{2} .
$$

For highly charged colloids and typical salt conditions, the corresponding density dependence is shown in Fig. 9, while the effective charge (deduced from the condition $Z_{\text {eff }}=Z_{\text {back }}$ ) is displayed in Fig. 10. When $\eta \rightarrow 0$, both quantities coincide with the infinite dilution limit of the traditional PB theory, as they should. The increase of $\kappa$ with the density of colloids reflects the increasing importance of counterion screening. The effective charge shows a nonmonotonous behavior with respect to density.

To compute the osmotic pressure, we subtract the reservoir pressure $\left(2 c_{s} k T\right)$ from expression (6). Moreover, it should be remembered that such a relation only provides the ionic contribution to the pressure. In the presence of salt and at low colloidal density this contribution becomes smaller

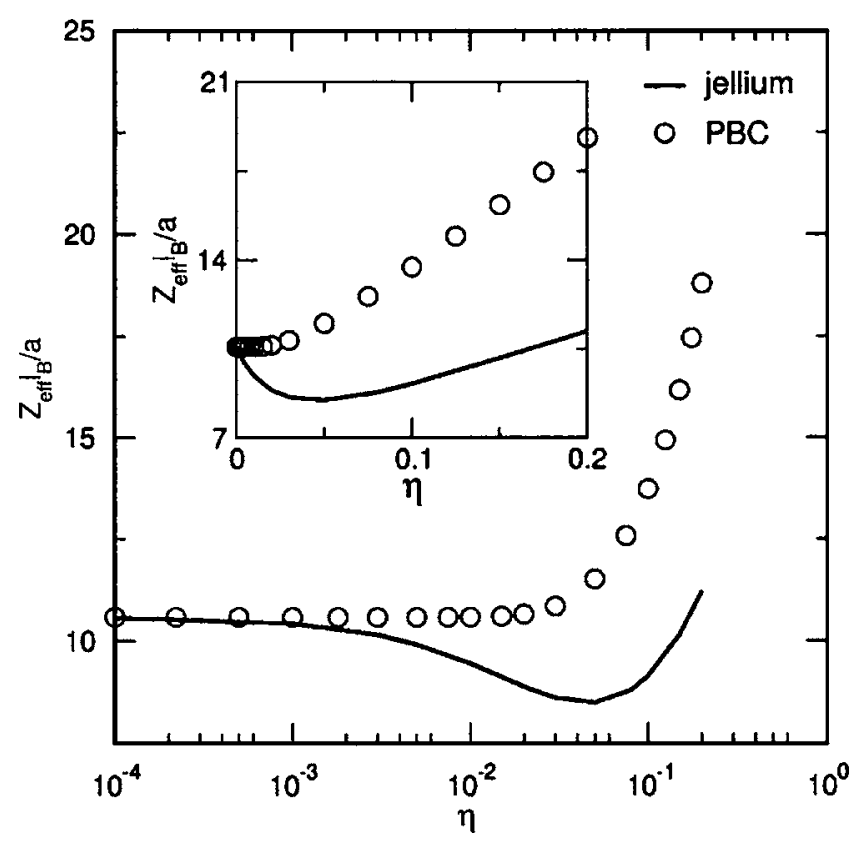

FIG. 10. The effective charge for spheres as a function of volume fraction within the $\mathrm{PBC}$ and the renormalized jellium model for $\kappa_{\mathrm{res}} a=1$ in the saturation regime $Z_{\text {bare }} \rightarrow \infty$. The inset shows the same quantity on a linear scale.

than the colloidal one. The vanishing of the microion contribution to pressure is exponential in the cell model, while it is algebraic for the jellium. Both models should then strongly disagree in the low density limit. To mimic the colloidal contribution, we add the ideal gas term $\rho k T$ to (6), so that the resulting osmotic pressure reads

$$
\beta \Pi=\rho+\sqrt{Z_{\mathrm{eff}}^{2} \rho^{2}+4 c_{s}^{2}}-2 c_{s}
$$

In the no salt case, addition of the ideal term is irrelevant since it is always much smaller than the microionic one, provided that $Z_{\text {eff }}$ is large enough (this is the case for highly or even weakly charged colloids since $a \gg \ell_{B}$ ). Moreover, addition of the ideal gas term breaks the scaling form valid in the no salt case where $a^{2} \ell_{B} \beta P$ only depends on $\eta$ and reduced charge $Z_{\text {bare }} \ell_{B} / a$. We therefore show the osmotic pressure in Fig. 11 for two values of colloid radius, within both the PBC and renormalized jellium frameworks. Apart from the expected deviations at small densities, one observes compatible values at higher $\eta$.

There exist relatively little simulational data for the primitive model with salt, where the bare Coulomb interactions between all charged species - colloids and microionsare taken into account (with still an implicit solvent). A reference equation of state with salt is provided in Ref. 32, with the simplification of a Wigner-Seitz cell but explicit microions. The simulations were performed in canonical ensemble with fixed salt content. The amount of added salt is characterized by a ratio of the overall added cation charge to the overall macroion charge, $\beta_{L}=C_{+} /\left(Z_{\text {bare }} \rho\right)$. We compute the densities $C_{ \pm}$corresponding to a given salt content as discussed in Sec. IV. In Fig. 12 the osmotic pressure $\beta P / \rho_{t}$ is plotted as a function of $\beta_{L}$, where $\rho_{t}$ is the total density of 


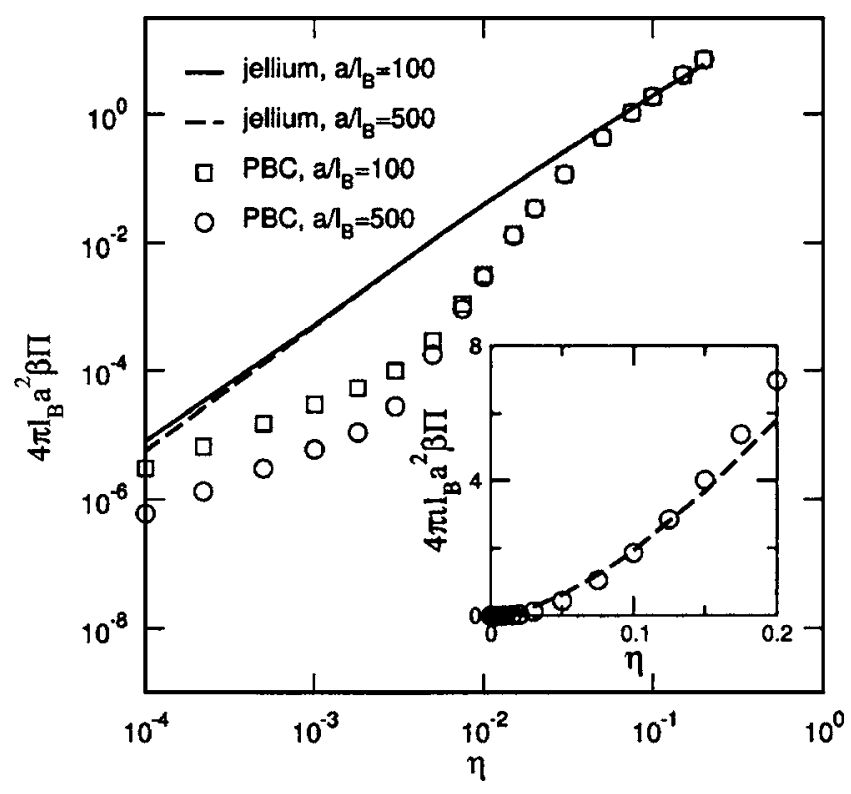

FIG. 11. The osmotic pressure as a function of volume fraction within the $\mathrm{PBC}$ and the renormalized jellium model in the saturation regime for $\kappa_{\mathrm{res}} a$ $=1$. The inset shows the same data on linear scale (spherical colloids).

ionic species. As in the case of salt-free suspensions the pressures calculated using the $\mathrm{PBC}$ and the renormalized jellium are in good agreement.

\section{B. Rodlike colloids}

For completeness, we briefly report here results for cylindrical geometry. Unlike the salt-free case where $\lambda_{\text {eff }}$ is a monotonic function of density, a minimum appears in the renormalized jellium curve shown in Fig. 13. The agreement between PBC and renormalized jellium at low $\eta$ signals the region where the system is salt dominated (the colloid den-

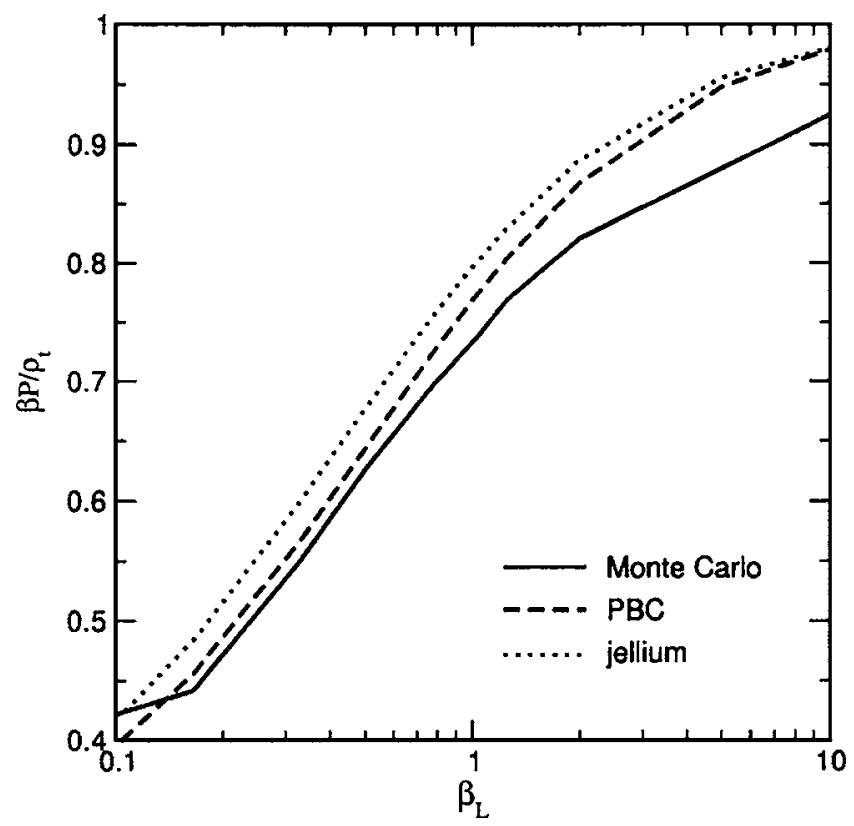

FIG. 12. Comparison of the PBC and the renormalized jellium equations of state with the one obtained in Ref. 32 from the Monte Carlo simulations. Here, the macroion volume fraction is $\eta=8.4 \times 10^{-3}$ while $Z_{\text {bare }} \ell_{B} / a$ $\simeq 21.45$.

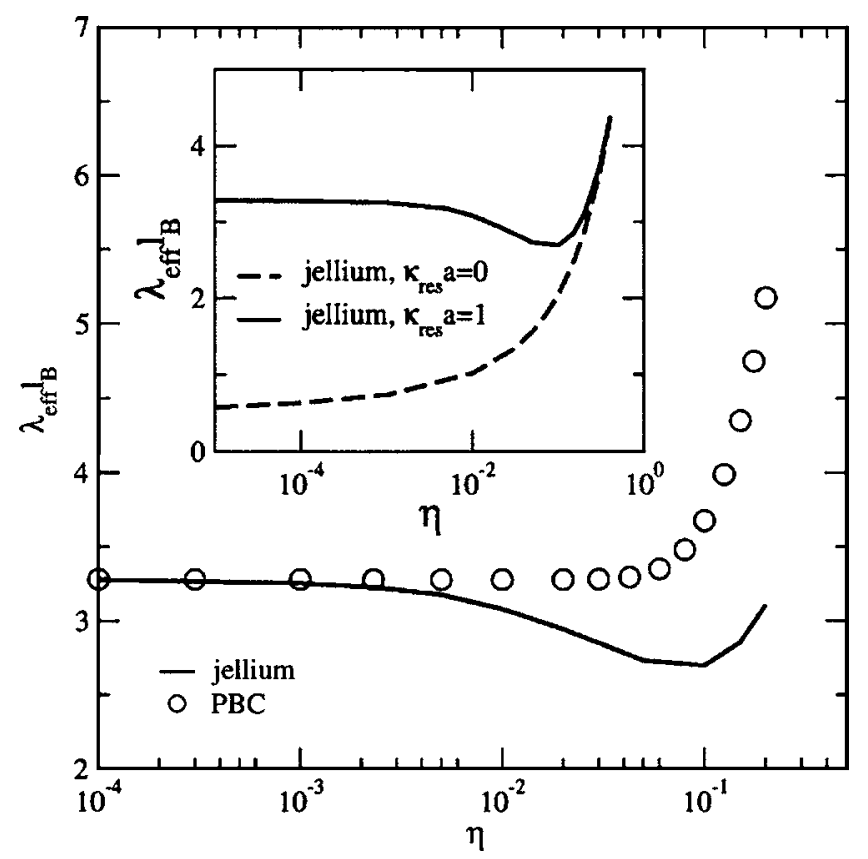

FIG. 13. The effective charge for highly charged cylindrical colloids (saturation regime) as a function of volume fraction within the $\mathrm{PBC}$ and the renormalized jellium model, for $\kappa_{\text {res }} a=1$. The inset shows appearance of a minimum in the presence of salt.

sity is too low and, consequently, counterions do not participate in the screening). Conversely, the inset indicates the density range where counterions do dominate: for $\eta>10^{-1}$, the results become independent of the reservoir ionic strength and coincide with those obtained in the no salt limit.

Finally, the pressure [Eq. (29)] for cylindrical colloids is given by

$$
4 \pi \ell_{B} a^{2} \beta \Pi=4 \eta \frac{\ell_{B}}{L}+\sqrt{\left(4 \lambda_{\mathrm{eff}} \ell_{B} \eta\right)^{2}+\left(\kappa_{\mathrm{res}} a\right)^{4}}-\left(\kappa_{\mathrm{res}} a\right)^{2} .
$$

Note that for infinite polyions $(L \rightarrow \infty)$, the first term on the right hand side of Eq. (30) vanishes. In Fig. 14 we plot the equation of state for polyions of $\lambda_{\text {bare }} \ell_{B}=2$. One should note a strong disagreement between the equations of state obtained using the renormalized jellium model and the PBC theory. In the case of cylindrical polyions the disagreement is exacerbated by the fact that the ideal gas contribution to the equation of state, Eq. (30), vanishes in the limit of $L \rightarrow \infty$ considered in this work. For small $\eta$, the behavior predicted by the renormalized jellium model is more realistic than that of the PBC.

\section{CONCLUSION}

Starting from a mean-field description in which a dispersion of $N$ spherical or rodlike polyions is treated using an $N$-body Poisson-Boltzmann theory, ${ }^{29}$ we have introduced the simplification of a homogeneous background to include the contribution of other colloids to the static field created by a tagged colloid. The charge of this background is consistently renormalized to coincide with the effective charge governing the far-field potential. This results in a nontrivial density de- 


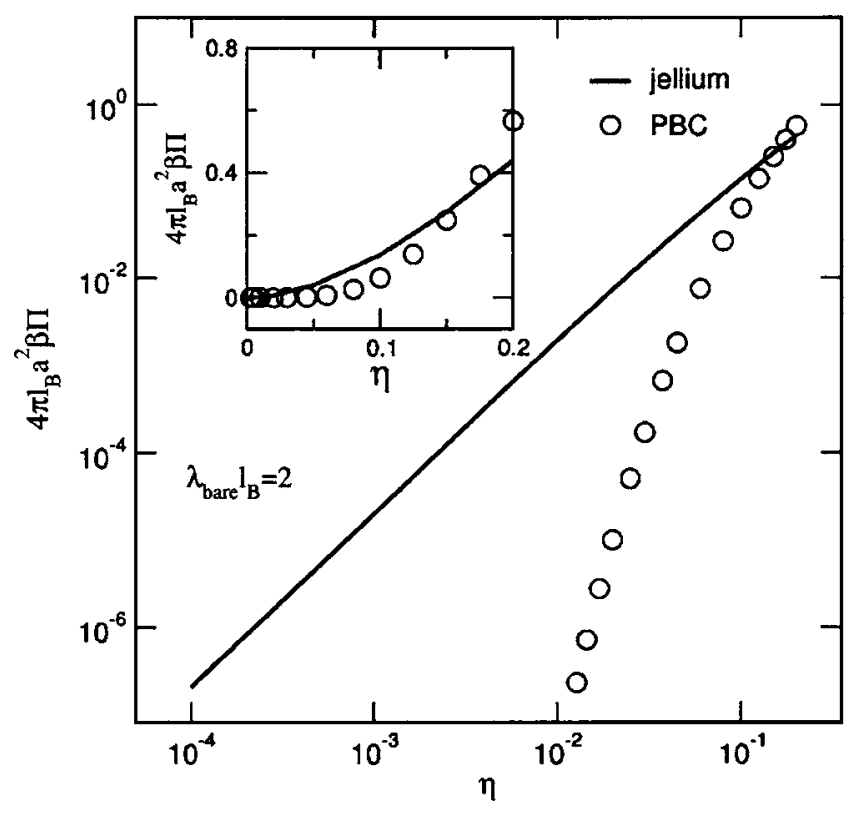

FIG. 14. Osmotic pressure as a function of volume fraction within the PBC and the renormalized jellium model for $\kappa_{\text {res }} a=1$ and $\lambda_{\text {bare }} l_{B}=2$. The inset shows the same data on linear scale (cylindrical colloids).

pendence of the effective colloidal charge, which directly enters into the equation of state through a simple analytical expression. The good agreement observed between the pressure calculated using the renormalized jellium and the Monte Carlo simulations confirms the relevance of the renormalized jellium model for theoretical and experimental purposes and provides an alternative to the Poisson-Boltzmann cell approach. Furthermore, we note that the effective charge calculated using the renormalized jellium model should be more relevant for the study of the effective interaction between the colloids than its Poisson-Boltzmann cell (PBC) counterpart. This is particularly the case since at finite colloidal density, the DLVO potential arises naturally within the jellium formalism, while it has to be introduced extraneously within the PBC. In this work, we have left untouched the question whether the pair potential calculated using jellium is a potential of mean force or an effective pair potential (following the terminology of Ref. 1). Further work is required to answer this question.

In a cylindrical geometry, the present approach implicitly subsumes an alignment between infinite rods-which is also a prerequisite for the analysis of Ref. 2-but contrary to the crystalline structure underlying the introduction of the cell model, we consider here systems with no positional order for the rods. As for spherical colloids the pressure in both approaches is in good agreement up to relatively high densities, whereas the effective charges differ significantly. We have also shown that the scenario for counterion condensation is similar to the cell picture.

Our approach, which is best suited to describe systems with low macroion densities, may be easily extended to the case of asymmetric electrolytes. One interesting aspect of the renormalized jellium is that the description of colloidal mixtures (macroions with different sizes and charges) appears to be as straightforward as for the monodisperse systems re- ported here. This is an important difference with the cell approach, which cannot be easily extended to such systems.

Among the possible refinements, it is possible to consider an inhomogeneous jellium with, again, renormalized charge. This should allow to extend the relevant range of densities where the model holds. Another interesting extension deals with the derivation of electrokinetic properties. Work along these lines is in progress.

\section{ACKNOWLEDGMENTS}

The financial support of Capes/Cofecub is gratefully acknowledged. We would also like to thank M. Deserno, J. Dobnikar, H. H. von Grünberg, R. Castañeda Priego, and L. Belloni for useful discussions.

${ }^{1}$ L. Belloni, J. Phys.: Condens. Matter 12, R549 (2000).

${ }^{2}$ T. Alfrey, Jr., P. W. Berg, and H. Morawetz, J. Polym. Sci. 7, 543 (1951); R. M. Fuoss, A. Katchalsky, and S. Lifson, Proc. Natl. Acad. Sci. U.S.A. 37, 579 (1951).

${ }^{3}$ S. Alexander, P. M. Chaikin, P. Grant, G. J. Morales, P. Pincus, and D. Hone, J. Chem. Phys. 80, 5776 (1984).

${ }^{4}$ J.-P. Hansen and H. Löwen, Annu. Rev. Phys. Chem. 51, 209 (2000).

${ }^{5}$ M. Deserno and C. Holm, in Proceedings of NATO Advanced Study Institute on Electrostatic Effects in Soft Matter and Biophysics, edited by C. Holm, P. Kekicheff, and R. Podgornik (Kluwer, Drodrecht, 2001), p. 27.

${ }^{6}$ S. Kuwabara,J. Phys. Soc. Jpn. 14, 527 (1959).

${ }^{7}$ H. Ohshima, Crit. Rev. Anal. Chem. 38, 139 (2004).

${ }^{8}$ Y. Levin, E. Trizac, and L. Bocquet, J. Phys.: Condens. Matter 15, S3523 (2003).

${ }^{9}$ Y. Levin, M. C. Barbosa, and M. N. Tamashiro, Europhys. Lett. 41, 123 (1998); A. Diehl, M. C. Barbosa, and Y. Levin, ibid. 53, 86 (2001).

${ }^{10}$ B. Beresford-Smith, D. Y. Chan, and D. J. Mitchell, J. Colloid Interface Sci. 105, 216 (1984).

${ }^{11}$ E. Trizac and Y. Levin, Phys. Rev. E 69, 031403 (2004).

${ }^{12}$ C. Haro-Pérez, M. Quesada-Pérez, J. Callejas-Fernández, R. Sabate, J. Estelrich, and R. Hidalgo-Álvarez, Colloids Surf., A 270, 352 (2005).

${ }^{13}$ C. Haro-Pérez, M. Quesada-Pérez, J. Callejas-Fernández, P. Schurtenberger, and R. Hidalgo-Álvarez, J. Phys.: Condens. Matter 18, L363 (2006).

${ }^{14}$ R. Castañeda-Priego, L. F. Rojas-Ochoa, V. Lobaskin, and J. C. MixtecoSánchez, e-print cond-mat/0608163.

${ }^{15}$ G. S. Manning, J. Chem. Phys. 51, 924 (1969); 51, 934 (1969); F. Oosawa, Polyelectrolytes (Dekker, New York, 1971).

${ }^{16}$ E. Trizac and G. Téllez, Phys. Rev. Lett. 96, 038302 (2006).

${ }^{17}$ Y. Levin, Rep. Prog. Phys. 65, 1577 (2002).

${ }^{18}$ M. Fushiki, J. Chem. Phys. 97, 6700 (1992); H. Löwen, J. P. Hansen, and P. A. Madden, ibid. 98, 3275 (1993).

${ }^{19}$ J. Dobnikar, Y. Chen, R. Rzehak, and H. H. von Grünberg, J. Chem. Phys. 119, 4971 (2003); J. Dobnikar, D. Haložan, M. Brumen, H. H. von Grünberg, and R. Rzehak, Comput. Phys. Commun. 159, 73 (2004).

${ }^{20}$ L. Belloni, Colloids Surf., A 140, 227 (1998).

${ }^{21}$ E. Trizac, L. Bocquet, and M. Aubouy, Phys. Rev. Lett. 89, 248301 (2002).

${ }^{22}$ L. Bocquet, E. Trizac, and M. Aubouy, J. Chem. Phys. 117, 8138 (2002).

${ }^{23}$ More precisely, it is the product $n_{i}^{0} \exp \left(-\beta z_{i} e \varphi_{\infty}\right)$ which is physically relevant. One may always choose $\varphi_{\infty}=0$, modulo a proper redefinition of the prefactors $n_{i}^{0}$. This convention is convenient for salt-free systems but has not been adopted in practice in the presence of added salt. In addition, Eq. (5) appears to be an effective electroneutrality condition, which does not coincide with the physical one. This aspect is discussed at the beginning of Sec. IV.

${ }^{24}$ E. Trizac, M. Aubouy, L. Bocquet, and H. H. von Grünberg, Langmuir 19, 4027 (2003).

${ }^{25}$ We emphasize that not all trial values of $\phi(\widetilde{R})$ lead to a solution. For a given $\widetilde{R}$, there indeed exists a critical threshold $\phi^{\text {sat }}(\widetilde{R})$ beyond which no solution can be found. For small $\phi(\widetilde{R})$ [i.e., $\phi(\widetilde{R}) \ll \phi^{\text {sat }}(\widetilde{R})$ ], there is a 
linear relationship between $Z_{\text {bare }}$ and $\phi(\widetilde{R})$, but when $\phi(\widetilde{R})$ approaches $\phi^{\text {sat }}(\tilde{R})$ from below, the bare charge diverges. This is a consequence of the phenomenon of effective charge saturation (Refs. 22 and 26) that is ubiquitous in mean-field treatments.

${ }^{26}$ G. Téllez and E. Trizac, Phys. Rev. E 68, 061401 (2003).

${ }^{27}$ M. Gueron and G. Weisbuch, Biopolymers 19, 353 (1980).

${ }^{28}$ B. O’Shaughnessy and Q. Yang, Phys. Rev. Lett. 94, 048302 (2005).
${ }^{29}$ This mean-field approach discards correlations between microions, that become prevalent at large electrostatic couplings (see Ref. 30). In a solvent such as water at room temperature, PB theory nevertheless provides a good description of monovalent ion systems, see, e.g., Ref. 31 or 17.

${ }^{30}$ A. Y. Grosberg, T. T. Nguyen, and B. I. Shklovskii, Rev. Mod. Phys. 74, 329 (2002).

${ }^{31}$ M. Deserno, C. Holm, and S. May, Macromolecules 33, 199 (2000).

${ }^{32}$ V. Lobaskin and K. Qamhieh, J. Phys. Chem. B 107, 8022 (2003). 